\title{
Stochastic Delay Analysis of Multi-services in Power Communication Networks
}

\author{
Yang Zhou1, Li Yu², Juhong Tian²*, Chao Luo ${ }^{2}$ \\ ${ }^{1}$ State Grid Jiangxi Electric Power Corporation Information Communication Branch, No.666, East Hubin RD, \\ Qingshanhu District, Nanchang City, Jiangxi Province, 330000, China. \\ 2 School of Electronic Information and Communication, Huazhong University of Science and Technology, \\ 1037 Luoyu RD, Hongshan District, Wuhan City, Hubei Province, 430074, China.
}

* Corresponding author. Tel.: +86-027-87792092; email: jhtian123@gmail.com

Manuscript submitted February 25, 2016; accepted April 26, 2016.

doi: 10.17706/ijcce.2016.5.6.409-418

\begin{abstract}
Various kinds of services transmitted over power communication networks are the key to make the power systems maintain normal operation. These various kinds of services may require different quality of service (QoS) guarantees, and scheduling mechanism is an efficient means to meet such requirement. In this study, we give a detailed introduction to several scheduling policies. To accurately evaluate the impacts of scheduling on the performance of services, based on stochastic network calculus, we derive the stochastic delay bounds of different types of services under the introduced scheduling policies.
\end{abstract}

Key words: Power communication networks, QoS, scheduling, Stochastic network calculus.

\section{Introduction}

The power communication networks are aimed to ensure the security and stability of the power system. It is the foundation of the electric power dispatching automation and one of most important infrastructures in the power system. With the development of smart grids, there has been a dramatic increase of the service data to be transferred in the power communication networks. Such growth drives a demand for upgrading the current power communication networks architecture, and harnesses information and communication technology in the areas of sensing, monitoring, controlling and computing to improve its greenness, efficiency, security, sustainability and stability [1], [2].

As a key technology for the next generation power communication network [3], packet transport network (PTN) can provide a reliable aggregation and transport infrastructure for any service type [4]. There are many types of services in power communication networks, such as dispatching telephone, device monitoring, and video conference and so on. Different types of services may require different levels of QoS guarantees, which means the power communication networks need to provide a QoS mechanism to meet different service demands [5]. To support this demand, PTN is evolving to provide IP transport and packet services which, with queue scheduling [6]-[8], will realize a flexible service guarantee [9] in power communication network.

In this paper, we classify the services of power communication networks into three types, each of which has a different level of QoS requirement. In addition, we introduce three kinds of scheduling algorithms [10], [11]: The queue properties of these scheduling algorithms are studied by stochastic network calculus 
[12]-[15], which can model all rate-based packet schedulers with a simple and elegant rate latency service curve [16]. Based on stochastic network calculus, we provide a unified treatment to a variety of problems arising in packet networks offering guaranteed service, and analyze the stochastic delay bounds of the three types of services under three kinds of scheduling algorithms. The derived results may provide insight on the design and service guarantee of power communication networks.

\section{Background}

\subsection{QoS Mechanisms in PTN}

QoS mechanism is one of the most important technologies in PTN. PTN provides QoS guarantees through three aspects: control plane, data plane and management plane. In data plane, it usually includes the following mechanisms: traffic classification, traffic regulation, congestion avoidance, queueing scheduling and traffic shaping, as shown in Fig. 1.

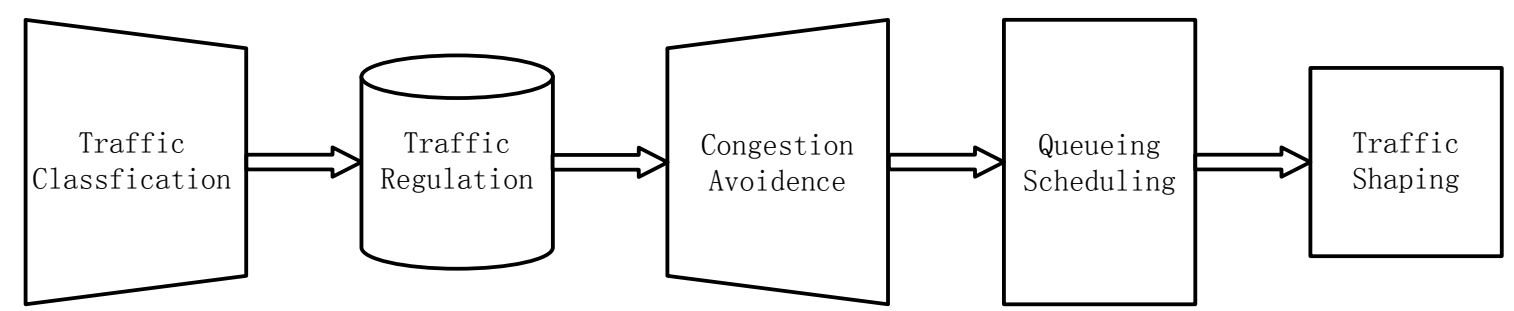

Fig. 1. Supported QoS Mechanisms in PTN.

- $\quad$ Traffic Classification

In traffic classification, flows are classified into different sets according to their QoS demands, which means flows with the same QoS requirements will be assigned to the same flow set.

- Traffic Regulation

Traffic regulation checks the flow whether conforms to the pre-negotiated policies, if not, the packets of the flow will be discarded.

\section{- $\quad$ Congestion Avoidance}

When the network load exceeds the network capacity, congestion will occur. A typical congestion avoidance mechanism is to drop some packets in the buffer.

- $\quad$ Queueing Scheduling

Queueing scheduling is one of the core mechanisms to provide QoS guarantees, and it enables different flows to get services of different levels.

- $\quad$ Traffic Shaping

Traffic shaping is employed to optimize or guarantee performance, to improve latency, and to increase usable bandwidth for some kinds of packets by delaying other kinds.

In this paper, we focus on exploring the impacts of queue scheduling policies on the performance guarantees. Our analysis is based on stochastic network calculus theory, and some basics of this theory are introduced in the following part of this section.

\subsection{Network Calculus Preliminaries}

We use $A(t), S(t)$ and $A^{*}(t)$ to denote the arrival process, the service process and the departure process in time interval $(0, t]$, respectively. $B(t)$ and $D(t)$ represent the queue length in the buffer and the delay at time $t$. We also denote $A(s, t)=A(t)-A(s), S(s, t)=S(t)-S(\mathrm{~s})$ and $A^{*}(\mathrm{~s}, t)=A^{*}(t)-A^{*}(s)$. Conventionally, we assume $A(t), S(t)$ and $A^{*}(t)$ have zero value at $t=0$. 
We denote $\mathscr{F}$ as the set of non-negative increasing function, in which each element function $f(\cdot)$ satisfies the following equation:

$$
\mathscr{F}=\{f(\cdot): \forall 0 \leq x \leq y, 0 \leq f(x) \leq f(y)\} .
$$

We denote $\overline{\mathscr{F}}$ as the set of non-negative increasing function, in which each element function $f(\cdot)$ satisfies the following equation:

$$
\overline{\mathscr{F}}=\{f(\cdot): \forall 0 \leq x \leq y, 0 \leq f(y) \leq f(x)\} .
$$

The following two inequalities hold for any non-negative functions $X(t)$ and $Y(t)$, and the proof of the following two inequalities can be referred in [3].

$$
\begin{aligned}
& \sup _{0 \leq s \leq t}[X(s)+Y(s)] \leq \sup _{0 \leq s \leq t} X(s)+\sup _{0 \leq s \leq t} Y(s), \\
& \inf _{0 \leq s \leq t}[X(s)-Y(s)] \geq \inf _{0 \leq s \leq t} X(s)-\sup _{0 \leq s \leq t} Y(s) .
\end{aligned}
$$

Min-plus algebra is the mathematical basic of network calculus. For given functions $f$ and $g$, the operations defined under min-plus algebra are as follows [1].

- The min-plus convolution of $f$ and $g$ is

$$
f \otimes g(t)=\inf _{0 \leq s \leq t}[f(s)+g(t-s)]
$$

- The min-plus de-convolution of $f$ and $g$ is

$$
f \oslash g(t)=\sup _{s \geq 0}[f(t+s)-g(s)]
$$

As mentioned above, $A(t)$ and $S(t)$ are used to denote the cumulative arrival process and the cumulative service process, and they are modeled as stochastic arrival curves and stochastic service curves in the analysis. Different models of stochastic arrival curves and stochastic service curves have been introduced in [7]. Our analysis is based on virtual-backlogged-centric stochastic arrival curves and virtualbacklogged-centric stochastic strict service curves, and their definitions are as follows.

Definition 1. A flow is said to have a virtual-backlog-centric stochastic arrival curve $\alpha \in$ F, with bounding function $f \in \overline{\mathscr{F}}$, denoted by $A(t) \sim_{v b}\langle f, \alpha\rangle$, if for all $t \geq 0$ and all $x \geq 0$, the following inequality holds

$$
P\left\{\sup _{0 \leq s \leq t}\{A(s, t)-\alpha(t-s)\}>x\right\} \leq f(x) .
$$

Definition 2. A system is said to be a virtual-backlog stochastic strict server providing service curve $\beta(t)$ with bounding function $g(x) \in \bar{F}$, denoted by $S \sim_{v b s s c}\langle g, \beta\rangle$, if during any period $(s, t]$, the amount of service $S(s, t)$ provided by the system satisfies 


$$
P\left\{\sup _{0 \leq s \leq t}[\beta(t-s)-S(s, t)]>x\right\} \leq g(x)
$$

for any $x \geq 0$.

\section{Stochastic Delay Bound Analysis}

In this section, we mainly discuss the delay bounds of different services under respective scheduling mechanisms in PTN-based power communication networks. There are many types of services in power communication network, including three typical types: scheduling voice service, real-time data service and monitoring service. For convenience, let $\mathrm{A}, \mathrm{B}$, and $\mathrm{C}$ denote these three services, respectively. Usually, $\mathrm{A}$ needs the highest level of QoS guarantees, and $\mathrm{B}$ comes second. Assume service $A, B$ and $C$ satisfy $A_{A} \sim\left\langle f_{A}, \alpha_{A}\right\rangle, A_{B} \sim\left\langle f_{B}, \alpha_{B}\right\rangle$ and $A_{C} \sim\left\langle f_{C}, \alpha_{C}\right\rangle$, respectively. We also assume the server satisfies $S \sim\langle g, \beta\rangle$.

\subsection{FIFO (First In, First Out) Scheduling Policy}

FIFO is the simplest priority based scheduling algorithm. It is the foundation of other scheduling mechanisms and has been widely used in current Internet. FIFO operates on strict "first come, first serve" basis and provides basic store-and-forward function (see Fig. 2).

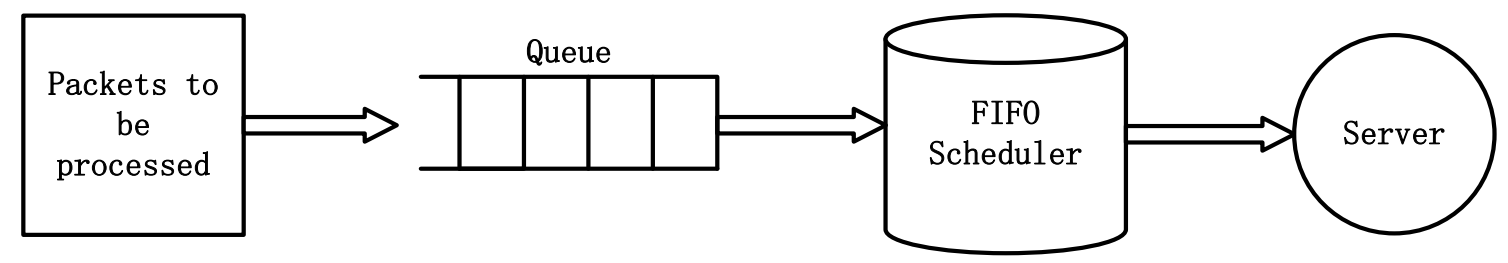

Fig. 2. A FIFO scheduling system.

As the packets in the queue tail may come from service $A, B$ and $C$ and the scheduler operates on "first come, first serve" basis, $A, B$ and $C$ have a similar delay. Therefore, we can regard the three types of service as one. According to the aggregation property [10], the combined service satisfies $A \sim\langle f, \alpha\rangle$, where $f(x)=f_{A} \otimes f_{B} \otimes f_{C}(x)$ and $\alpha=\alpha_{A}+\alpha_{B}+\alpha_{C}$.

Based on the properties mentioned in section 2, we derive the delay bound of the combined service as

$$
P\{D(t)>x\} \leq f \otimes g\left(\inf _{s \geq 0}[\beta(s+x)-\alpha(s))\right.
$$

The delay of a packet is the difference between the timestamp when the packet arrives at the system and the timestamp when the packet leaves the system. Hence, the delay for the packet arrives at the system at time $t$ can be expressed as.

$$
D(t)=\inf \left\{\tau \geq 0: A(t) \leq A^{*}(t+\tau)\right\}
$$

This expression means for any $x \geq 0$, if $D(t)>x$, there must be $A(t)>A^{*}(t+\tau)$. Therefore,

$$
P\{D(t)>x\} \leq P\left\{A(t)>A^{*}(t+x)\right\}
$$

Since 


$$
\begin{aligned}
A(t)-A^{*}(t+x) \\
=A(t)-A \otimes S(t+x) \\
=A(t)-\inf _{0 \leq s \leq t+x}[A(s)+S(s, t+x)] \\
=\sup _{0 \leq s \leq t+x}[A(s, t)-S(s, t+x)] \\
=\sup _{0 \leq s \leq t+x}[A(s, t)-\alpha(t-s)+\beta(t+x-s)-S(s, t+x)+\alpha(t-s)-\beta(t+x-s)] \\
\leq \sup _{0 \leq s \leq t+x}[A(s, t)-\alpha(t-s)]+\sup _{0 \leq s \leq t+x}[\beta(t+x-s)-S(s, t+x)]+\sup _{0 \leq s \leq t+x}[\alpha(t-s)-\beta(t+x-s)] \\
=\sup _{0 \leq s \leq t+x}[A(s, t)-\alpha(t-s)]+\sup _{0 \leq s \leq t+x}[\beta(t+x-s)-S(s, t+x)]-\inf _{v \geq 0}[\beta(u+x)-\alpha(u)]
\end{aligned}
$$

Then we obtain the delay bound as

$$
\begin{aligned}
& P\{D(t)>x\} \\
& \leq P\left\{A(t)-A^{*}(t+x)>0\right\} \\
& \leq P\left\{\sup _{0 \leq s \leq t+x}[A(s, t)-\alpha(t-s)]+\sup _{0 \leq s \leq t+x}[\beta(t+x-s)-S(s, t+x)]-\inf _{v \geq 0}[\beta(u+x)-\alpha(u)>0\}\right. \\
& =P\left\{\sup _{0 \leq s \leq t+x}[A(s, t)-\alpha(t-s)]+\sup _{0 \leq s \leq t+x}[\beta(t+x-s)-S(s, t+x)]>\inf _{v \geq 0}[\beta(u+x)-\alpha(u)\}\right. \\
& \leq f \otimes g\left(\inf _{v \geq 0}[\beta(u+x)-\alpha(u))\right.
\end{aligned}
$$

\subsection{PQ (Priority Queueing) Scheduling Policy}

A priority queueing scheduler maintains a series of queues. Only when all the packets in the queues with higher priorities have been processed, the packets in the queues with lower priorities could get service (see Fig. 3).

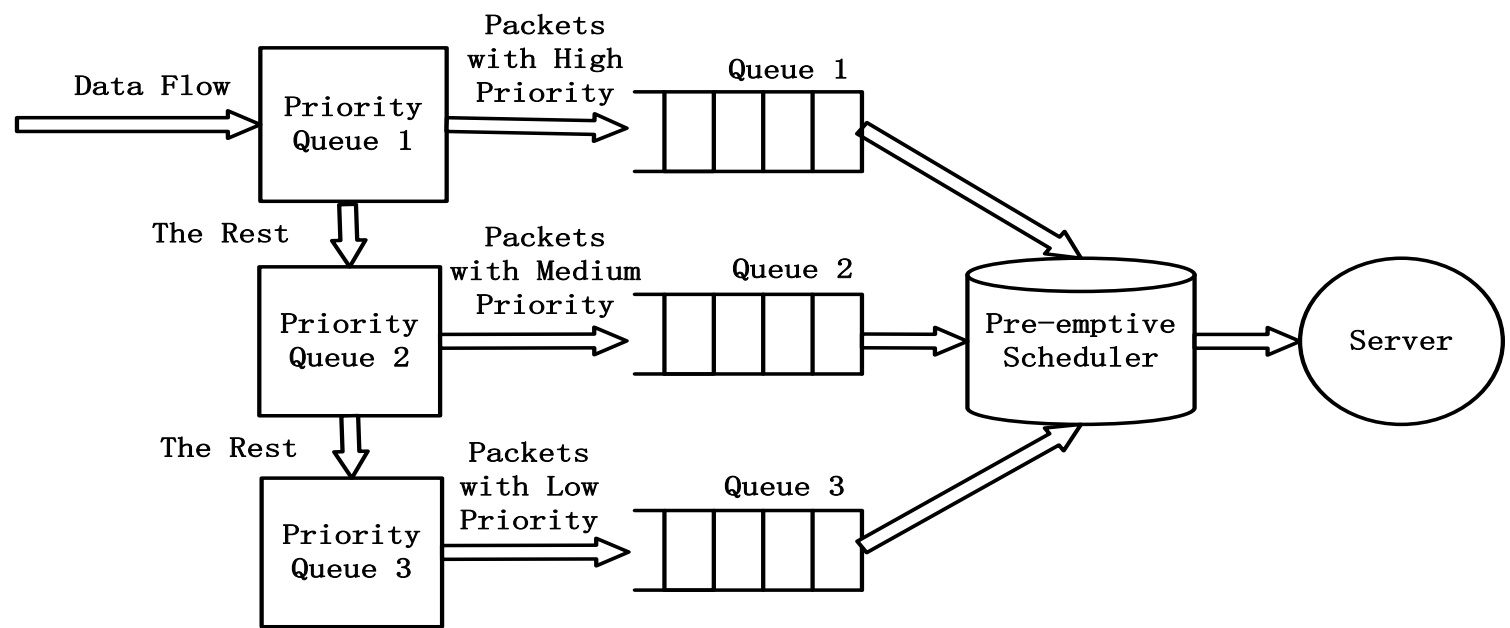

Fig. 3. A PQ scheduling system.

Assume that service $A$ has a higher priority, $B$ has a medium priority and $C$ has a lower priority. For service $A$, it suffers no competition from service $B$ and $C$, which means service $B$ and service $C$ can be seen as not existed. Hence, the delay bound of service $A$ is 


$$
P\left\{D_{A}(t)>x\right\} \leq f_{A} \otimes g\left(\underset{s \geq 0}{\inf }\left[\beta(s+x)-\alpha_{A}(s)\right) .\right.
$$

For service $B$, it can only get service when all the packets of service $A$ have been processed. At the same time, it suffers no competition from service $C$. We can regard service $A$ and service $B$ combined as service $B$, and $B$ and $B^{\prime}$ has the same delay bound. Hence, the delay bound of service $B$ is

$$
P\left\{D_{B}(t)>x\right\} \leq f_{B^{\prime}} \otimes g\left(\inf _{s \geq 0}\left[\beta(s+x)-\alpha_{B^{\prime}}(s)\right),\right.
$$

where $f_{B^{\prime}}(x)=f_{A} \otimes f_{B}(x)$ and $\alpha_{B^{\prime}}=\alpha_{A}+\alpha_{B}$.

For service $C$, we can regard services $A, B$ and $C$ combined as $C$. Similar as the analysis for services $B, C$ and $C^{\prime}$ has the same delay bound

$$
P\left\{D_{C}(t)>x\right\} \leq f_{C^{\prime}} \otimes g\left(\inf _{s \geq 0}\left[\beta(s+x)-\alpha_{C^{\prime}}(s)\right),\right.
$$

where $f_{C^{\prime}}(x)=f_{A} \otimes f_{B} \otimes f_{C}(x)$ and $\alpha_{C^{\prime}}=\alpha_{A}+\alpha_{B}+\alpha_{C}$.

\subsection{CQ (Custom Queueing) Scheduling Policy}

CQ could support 17 queues at most, numbered from 0 to 16 . Queue 0 is the queue with highest priority, only when the packets in queue 0 have been processed, the scheduler allocates service to other queues. CQ adopts Round Robin scheduling algorithm for queue 1 to queue 16, and it pre-configures the amount of packets to be processed for each queue. The Fig. 4 shows the schematic of CQ scheduling system.

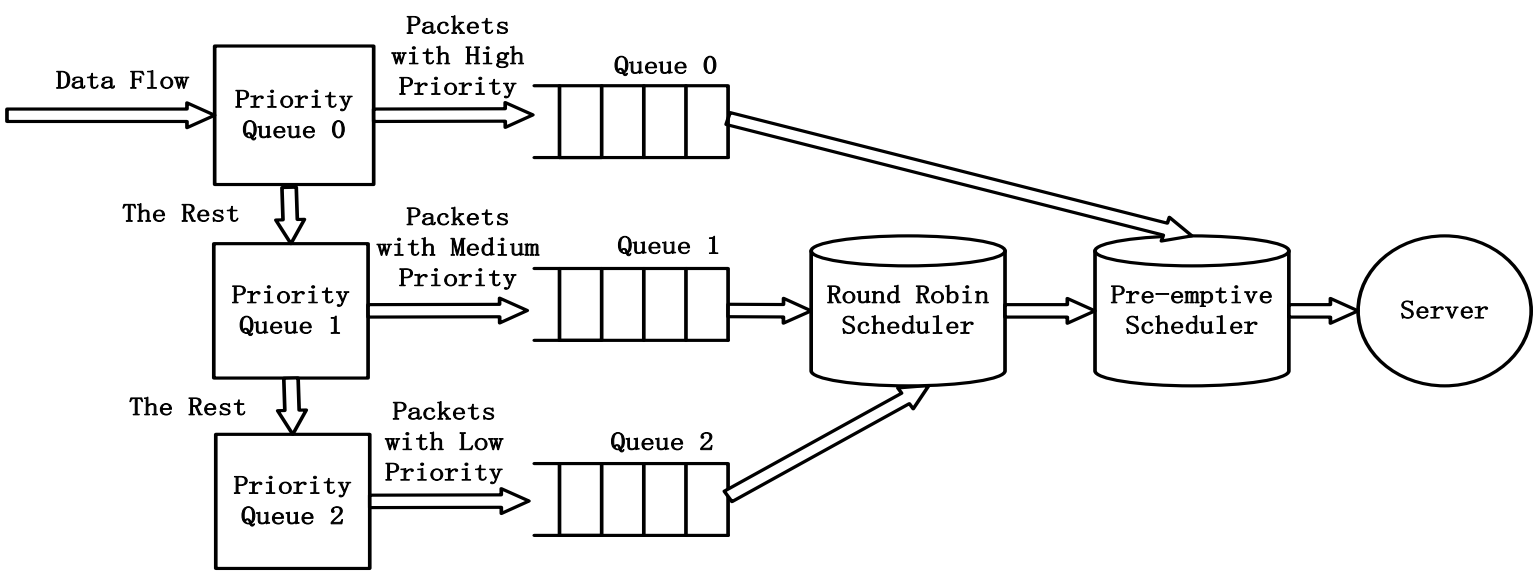

Fig. 4. A CQ scheduling system.

Let service $A$ have the highest priority, service $B$ and $C$ are pre-configured with the amount of packets by $b$ and $c$. For service $A$, service $B$ and service $C$ can be ignored, and the delay bound is

$$
P\left\{D_{A}(t)>x\right\} \leq f_{A} \otimes g\left(\inf _{s \geq 0}\left[\beta(s+x)-\alpha_{A}(s)\right) .\right.
$$

For service $B$ and service $C$, they can get the leftover while the handled service $A$ has been handled. Let $S^{\prime}$ denote the leftover service, then $S^{\prime}=S-A$.

As $S^{\prime}=S-A$ then 


$$
\begin{aligned}
& \sup _{0 \leq s \leq t}\left[\beta^{\prime}(t-s)-S^{\prime}(s, t)\right] \\
& =\sup _{0 \leq s \leq t}\left[\beta(t-s)-\alpha_{A}(t-s)-S^{\prime}(s, t)+A_{A}(s, t)\right] \\
& \leq \sup _{0 \leq s \leq t}[\beta(t-s)-S(s, t)]+\sup _{0 \leq s \leq t}\left[A_{A}(s, t)-\alpha_{A}(t-s)\right] \\
& \leq g \otimes f_{A}(x) .
\end{aligned}
$$

Then $S^{\prime}$ has a stochastic service curve

$$
S^{\prime} \sim\left\langle g^{\prime}, \beta^{\prime}\right\rangle
$$

where $\beta^{\prime}(t)=\beta(t)-\alpha_{A}(t)$ and $g^{\prime}(x)=g \otimes f_{A}(x)$.

According to the scheduling mechanism of CQ, service $B$ and service $C$ at least get the leftover service with the ratio of $\lambda_{B}$ and $\lambda_{C}$, where $\lambda_{B}=b / b+c$ and $\lambda_{C}=c / b+c$. Let $S_{B}=\lambda_{B} \cdot S^{\prime}$ and $S_{C}=\lambda_{C} \cdot S^{\prime}$, then

$$
\begin{aligned}
& S_{B} \sim\left\langle g_{B}, \beta_{B}\right\rangle, \\
& S_{C} \sim\left\langle g_{C}, \beta_{C}\right\rangle,
\end{aligned}
$$

where $g_{B}(x)=g^{\prime}\left(x / \lambda_{B}\right), \quad g_{C}(x)=g^{\prime}\left(x / \lambda_{C}\right), \quad \beta_{B}(t)=\lambda_{B} \beta^{\prime}(t)$ and $\beta_{C}(t)=\lambda_{C} \beta^{\prime}(t)$.

According to the Definition, we have

$$
P\left\{\sup _{0 \leq s \leq t}\left[\beta^{\prime}(t-s)-S^{\prime}(s, t)\right]>x\right\} \leq g^{\prime}(x) .
$$

Let the left side of (14) by coefficient $\lambda_{B}$

$$
P\left\{\sup _{0 \leq s \leq t}\left[\lambda_{B} \beta^{\prime}(t-s)-\lambda_{B} S^{\prime}(s, t)\right]>\lambda_{B} x\right\} \leq g^{\prime}(x) .
$$

Let $\beta_{B}(t-s)=\lambda_{B} \beta^{\prime}(t-s), \quad S_{B}(s, t)=\lambda_{B} S^{\prime}(s, t)$ and $x_{B}=\lambda_{B} x$ in (15), we get

$$
P\left\{\sup _{0 \leq s \leq t}\left[\beta_{B}(t-s)-S_{B}(s, t)\right]>x_{B}\right\} \leq g^{\prime}\left(x_{B} / \lambda_{B}\right)
$$

Then

$$
\begin{aligned}
& S_{B} \sim\left\langle g_{B}, \beta_{B}\right\rangle, \\
& S_{C} \sim\left\langle g_{C}, \beta_{C}\right\rangle,
\end{aligned}
$$

where $g_{B}(x)=g^{\prime}\left(x / \lambda_{B}\right), \quad g_{C}(x)=g^{\prime}\left(x / \lambda_{C}\right), \quad \beta_{B}(t)=\lambda_{B} \beta^{\prime}(t)$ and $\beta_{C}(t)=\lambda_{C} \beta^{\prime}(t)$.

Then the delay bounds of service $B$ and service $C$ can be derived as

$$
P\left\{D_{B}(t)>x\right\} \leq f_{B} \otimes g_{B}\left(\inf _{s \geq 0}\left[\beta_{B}(s+x)-\alpha_{B}(s)\right),\right.
$$




$$
P\left\{D_{C}(t)>x\right\} \leq f_{C} \otimes g_{C}\left(\inf _{s \geq 0}\left[\beta_{C}(s+x)-\alpha_{C}(s)\right) .\right.
$$

From the analysis above, we can see that FIFO cannot provide differentiated services. PQ could ensure the service with high priority get sufficient service resources and good performances. However, when there is a burst of the service with higher priorities, the service with low priority may not get processed for a long time. That means the delay of the service with low priority may be large. CQ not only guarantees the service with high priority get sufficient service resources, but also can make sure other types of services obtain reasonable service resources in time. Since all the services in power communication networks should get differential QoS guarantees, CQ is the most suitable one among the three scheduling mechanisms.

\section{Validation of Stochastic Delay Bounds}

In this section, some simulations are conducted to verify our analysis above. The simulations are based on SimEvents, and the basic simulation model is shown in Fig. 5. According to different scheduling policies, the simulation models are also modified.

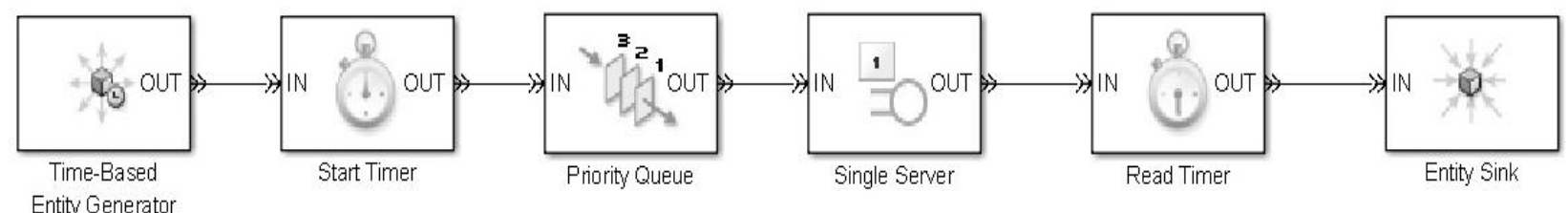

Fig. 5. The basic simulation model.

We assume the arrival processes of $A, B, C$ follow Poisson distributions, and the server provides service with a constant rate. The parameters can be referred to in Table 1.

Table 1. The Simulation Parameters

\begin{tabular}{lll}
\hline \hline Channels & Distribution & Parameters (Mbps) \\
\hline$A$ & Poisson & $\lambda_{A}=4$ \\
$B$ & Poisson & $\lambda_{B}=6$ \\
$C$ & Poisson & $\lambda_{C}=9$ \\
Service & Constant & $r=20$ \\
\hline \hline
\end{tabular}

According to Definition 1 and the property of Poisson process, for service $A$, we have

$$
P\{A(s, s+t)-\lambda t>x\}=P\{A(s+t)-A(s)>\lambda t+x\} \leq \sum_{n=\{x+\lambda t\rceil}^{\infty} \frac{e^{-\lambda t} \cdot(\lambda t)^{n}}{n !} .
$$

Then the stochastic arrival curve of service $A$ can be approximated by (20)

$$
P\{A(s, s+t)-4 t>x\} \leq e^{x-(4 t+x) \ln \frac{4 t+x}{4 t}} .
$$

The stochastic arrival curves of service B and C can be obtained in a similar way. For each scheduling policy of FIFO, PQ and CQ the simulations are conducted for a hundred times. Fig. 6 shows the theoretical and simulation delay bounds of service A, B, C under three scheduling policies. In particular, according to the previous analysis, under FIFO scheduling policy, services achieve the same theoretical delay bound, 
while the difference of the simulation delay bounds on the three services is quite small as shown in (a). As depicted in (b) and (c), service A has a similar delay bound which is in accordance with our previous analysis that service A suffers no competition from other services since it has the highest priority in PQ and CQ. From Fig. 6, we can also observe that the gaps between the theoretical results and simulation results are very small, which validates the accuracy of our analysis.

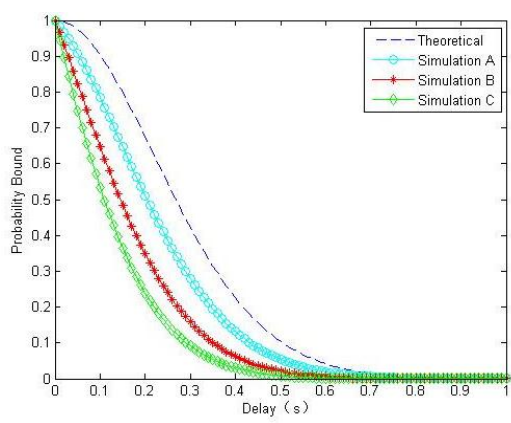

(a). FIFO;

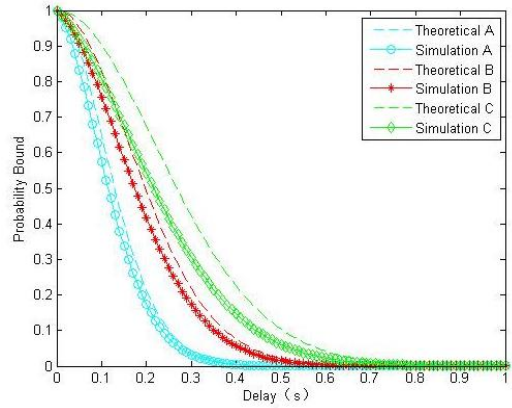

(b). PQ;

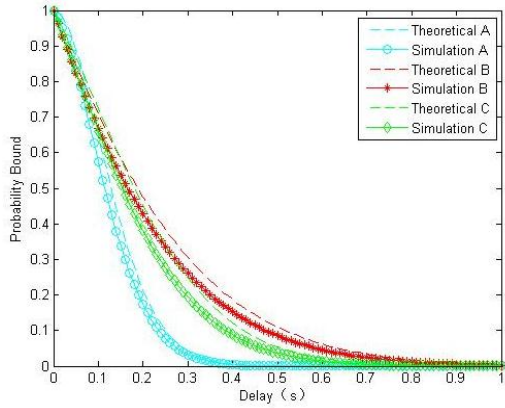

(c). CQ;

Fig. 6. The stochastic delay bounds under FIFO, PQ and CQ.

\section{Conclusion}

In power communication networks, various kinds of services require differentiated quality of service (QoS) guarantees, and scheduling mechanism is an efficient means to meet such requirement. In this paper, we introduce three kinds of scheduling policies into power communication networks and explore their performance bounds on different services. Based on stochastic network calculus, we accurately evaluate the impacts of scheduling on the performance of services, and derive the stochastic delay bounds of these services under the introduced scheduling policies. Based on our analysis, we show that CQ could not only provide the best QoS guarantees to the service with the highest priority, but also provide the customized resources to other services. Our results reveal that CQ is the most suitable scheduling policy for the service of power communication networks. By identifying the key impact factors of delay performance mathematically, our analysis may provide insight on the design of power communication networks.

\section{Acknowledgment}

This article is supported by National Natural Science Foundation of China (60972016) and Project of the Sci-tech Progress Foundation of the State Grid Jiangxi Province Power Company (NO.52183514008g).

\section{References}

[1] Farhangi, H. (2010). The path of the smart grid. IEEE Power Energy Magazine, 8(1), 18-28.

[2] Ipakchi, A., \& Albuyeh, F. (2009). Grid of the future. IEEE Power Energy Magazine, 7(2), 52-62.

[3] Luo, Z. C., Yu, J., Chang, J., et al. (2011). Modeling and simulation of distribution power communication traffic engineering based on PTN. Computer Science and Network Technology, 1654-1658.

[4] Feng, B., Fab, Q., \& Li, Y. (2012). Research on framework of the next generation power communication transmission network. Computer Science and Electronics Engineering, 414-417.

[5] Ke, J., Chang, J., Zong, R., \& Yu, J. (2011). Research on QoS of PTN-based power distribution communication network. Computer Science and Network Technology, 414-417.

[6] Faucheu, L. F., Wu, L., Davie, B., Davari, S., Vaananen, P., et al. (2002). Multi-protocol label switching (MPLS) support of differentiated services. RFC 3270.

[7] Blake, S., Black, D., Carlson, M., et al. (1998). An architecture for differentiated services. RFC 2475.

[8] Fidler, M., \& Rizk, A. (2015). A guide to the stochastic network calculus. IEEE Communications Surveys 
\& Tutorials, 17(1), 92-105.

[9] Rizk, A., \& Fidler, M. (2012). Non-asymptotic end-to-end performance bounds for networks with long range dependent FBM cross traffic. Computer Networks, 56(1), 127-141.

[10] Ke, J., Chang, J., et al. (2013). Shortest path finding problem in stochastic time-dependent road networks with stochastic first-In-first-out property. Intelligent Transportation Systems, 14, 414-417.

[11] Sudiharto, D. W., Yulianto, F. A., \& Arista, A. N. (2015). Comparative analysis of voice over internet protocol (VoIP) quality on priority queue (PQ) and class-based queue (CBQ) management system using link-sharing mechanism setting. Information and Communication Technology, 419-424.

[12] Liebeherr, J., Burchard, A., \& Ciucu, F. (2012). Delay bounds in communication networks with heavy-tailed and self-similar traffic. IEEE Transactions on Information Theory, 58(2), 1010-1024.

[13] Jiang, Y., \& Liu, Y. Stochastic Network Calculus. London, U.K.: Springer-Verlag.

[14] Mao, S., \& Panwar, S. S. (July 2006). A survey of envelope processes and their applications in quality of service provisioning. IEEE Communications Surveys \& Tutorials, 8(3), 2-20.

[15] Fidler, M. (2010). A survey of deterministic and stochastic service curve models in the network calculus. IEEE Communications Surveys \& Tutorials, 12(1), 59-86.

[16] Ciucu, F., \& Schmitt, J. (August 2012). Perspectives on network calculus-No free lunch but still good value. Proceedings of ACM SIGCOMM (pp. 311-322).

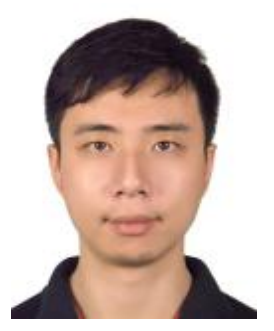

Yang Zhou was born in 1986, he received his B.S degree from Nanchang University in 2008 and received his M.S degree from Communication University of China in 2010. He is now engaged in engineer in Jiangxi power grid. His research interests include optical fiber communication and electric power data communication network fault location.

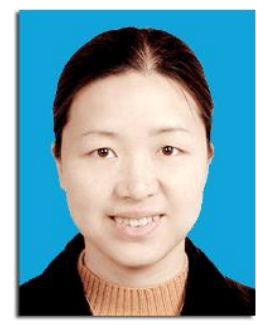

Li Yu was born in 1970, she is a professor, and she received her B.S, M.S, and Ph.D. degrees all from Huazhong University of Science and Technology in 1992, 1995, 1999 respectively.

She is now the dean of the Division of Communication and Intelligent Network, WNLO. Her research focuses on multimedia, wireless network, video coding decoding and many other related areas.

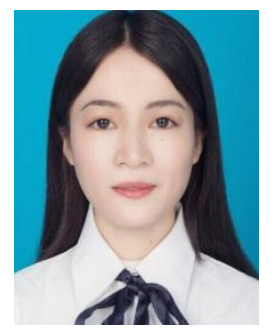

Juhong Tian was born in 1991, she received her BS degree from Hunan University in 2014. She is an M.S student in Huazhong University of Science and Technology since 2014. Her research focuses on data mining and stochastic network calculus.

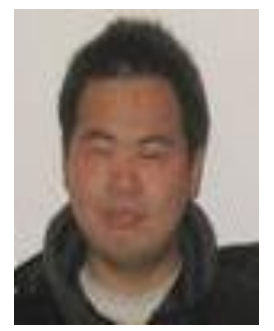

Chao Luo was born in 1985, he received his BS and MS degrees from Wuhan University of Technology in 2007, 2010 respectively. He is a Ph.D. candidate in Huazhong University of Science and Technology since 2010. His research focuses on stochastic network calculus. 